\title{
Mechanical Experiments about Pendulum Support of Vacuum Vessel W7-X
}

\author{
B. Missal 1), Dr. A. Cardella1), Dr. M. Schrader1), T. Koppe1), Dr. P. Friedrich2) \\ 1) Max-Planck-Institut für Plasmaphysik, EURATOM Association, Teilinstitut Greifswald, Wendelsteinstraße 1, \\ D-17491 Greifswald, Germany (email: bernd.missal@ipp.mpg.de, phone: +49-3834-88-2791, fax: +49-3834-88-2709)
}

\section{2) Universität Rostock, FB Maschinenbau und Schiffstechnik, A.-Einsteinstr. 2, 18051 Rostock, Germany}

\begin{abstract}
At the Max-Planck-Institut für Plasmaphysik (IPP) the new stellarator fusion experiment WENDELSTEIN 7-X (W7-X) is under construc-tion. Its ultimate goal is to verify that such stellarator magnetic confinement concept is a viable option for a demonstration fusion power-plant.

Its toroidal plasma (major diameter of $11 \mathrm{~m}$ ) is enclosed by a cryostat. The Outer Vessel (OV), ports and the Vacuum Vessel (VV) accumulate the cryostst together.

The main functions of the VV supports (VVS) are to withstand and transfer all vertical forces from the VV to the machine base and to allow horizontal movements simultaneously. Therefore pendulum support system was introduced. The paper will describe their design and the destinations and results of a test campaign (scale 1:1) at the University of Rostock.

vacuum vessel support, pendulum, spherical bearing
\end{abstract}

\section{Introduction}

At the Max-Planck-Institut für Plasmaphysik (IPP) in Greifswald, the new stellerator Wendelstein 7-X (W7-X) is under construction. Its toroidal plasma (major diameter of $11 \mathrm{~m}$ ) is directly enclosed by the Vacuum Vessel (VV). The vertical VV support system must fulfil 3 functions:

1. Withstand all the vertical loads

2. Vertical adjustment (by hydraulic cylinders)

3. Allow (passive) horizontal movement

Figure 1 shows a schematic cut through the cryostat where are shown the main components (especially one inner vertical support) and the movements.

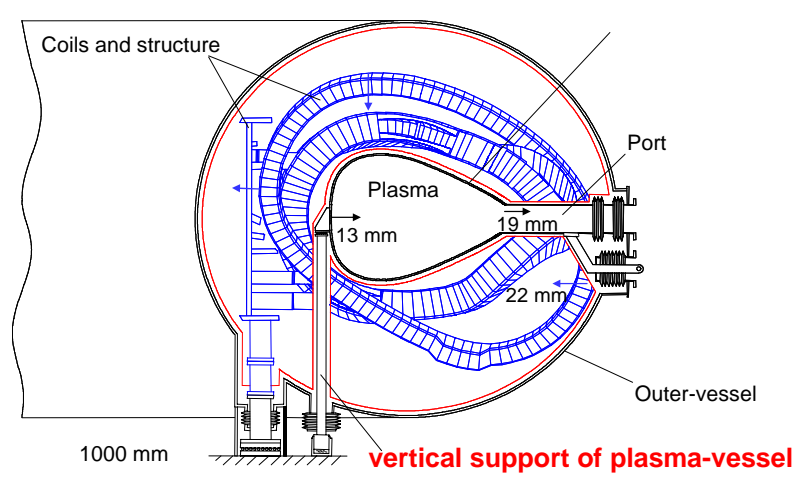

Fig. 1: Schematic view of whole cryostat
2. Values and Design of the vertical support system

The VV of W7-X consists of five uniform modules. Each of them must be supported and adjusted separately.

Therefore each PV module will be supported by three vertical supports which are of different lengths because of geometrical environment. Two supports are located on the inner side and the third is placed at the outer side of the VV. (Fig. 2)

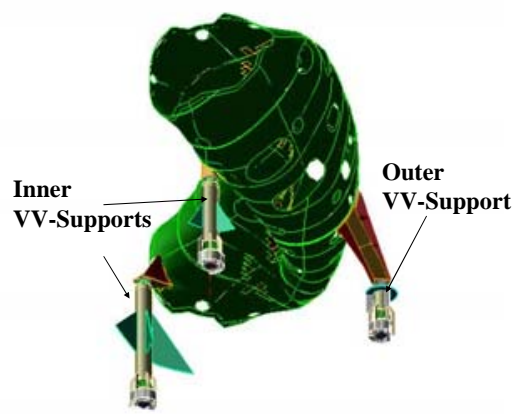

Fig. 2: Vacuum vessel module with the 3 vertical support-structures

The total number of VVS are then 15.

The total vertical load inclusive safety margin is $1300 \mathrm{kN}$. Conservatively it has been estimated, that this load is only distributed on 10 supports. Therefore every support has to withstand up to 130 $\mathrm{kN}$.

Because of thermal expansion and horizontal adjustment the system has to withstand 200 horizontal strokes in its life time.

Considering a large safety margin it was decided to calculate and test the supports with a number of 1000 full strokes.

The maximal shrinkage during operation is $10 \mathrm{~mm}$ and the expansion $20 \mathrm{~mm}$.

To withstand all mentioned vertical loads, allow horizontal movement and reduce friction forces the VV support has been designed as a pendulum support. (Fig. 3) 


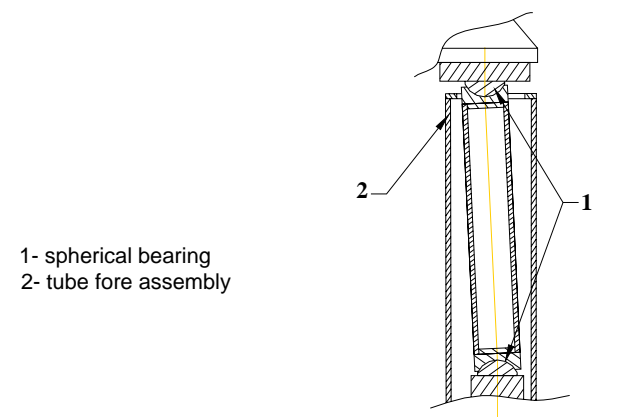

Fig. 3: Schematic view of the pendulum support

This design consists of vertical tubes with semi spherical bearings at their ends. The semi spherical bearing on the bottom is fixed, but the top of the support is free to rotate. The pendulums can withstand all mentioned vertical loads and allow the horizontal movements simultaneously. In order to avoid that the PV tilt, separate horizontal supports fix the PV horizontally.

\section{Test of the pendulum support system}

\subsection{Experimental objektives}

The goal was to find a convenient material and geometry of the hemisphere (ball/cup).

Therefore analyses and tests had to be performed. In order to prove that this pendulum support system has a low frictional resistance a series of experiments was developed, which were carried out at the University of Rostock.

At first the influence of the length of the pendulum support was examined. Secondly the required horizontal forces and respectively the equivalent friction factor were found.

The friction factor depends on lubrication. In the non-lubricated state, so-called Stick Slip Effects could occur. Vacuum compatible molybdenum disulphide has been employed. However, it is possible during the long time of the operation that the lubrication is partially removed. For this reason the main tests have been carried out in the worst case condition when the sliding parts are notlubricated.

\subsection{Experimental setup}

To fulfil original conditions 3 pendulum supports were produced and tested with the original dimensions. The University of Rostock provided a rugged strength frame. The design load of $130 \mathrm{kN}$ was applied with a force controlled hydraulic cylinder (Det. 4 in Fig. 4). The vertical force was constantly controlled with a load cell (2). The VV can shrink by $10 \mathrm{~mm}$ and extend by $20 \mathrm{~mm}$ during the different operations states. To impact this horizontal movements a second hydraulic cylinder (3) was used. This hydraulic cylinder is displacement controlled and the required force for those movements was measured with a load cell (1) too.

The rolling resistance of the roller bearing (5) was determined in a pre-test. The value was found negligible.

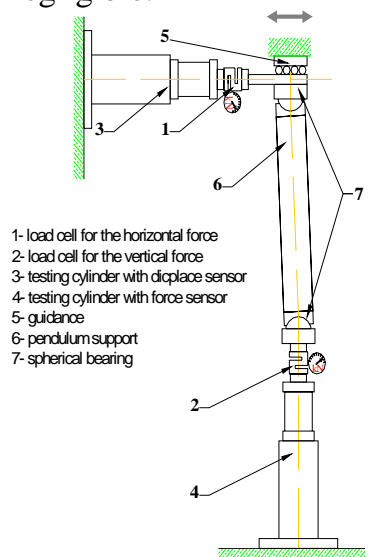

Fig. 4. Principle of test

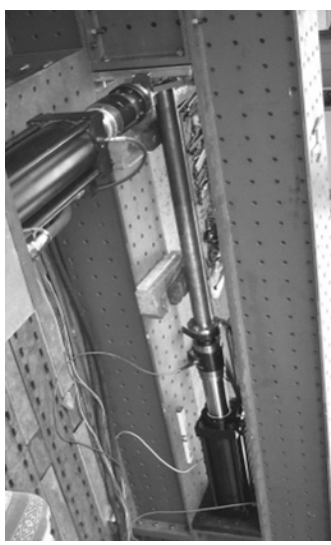

Fig. 5: Original of test

\subsection{Testing procedure and results}

Different test campaigns were performed.

To see the influence of the tests the geometry and roughness of the tubes, the balls and the cups where measured in the first test campaign before and after the tests. In all cases here where only very small changes. Therefore this was negligible.

After each test resulting dates were compared with the objectives. Than were created new modifications for the subsequent campaigns. All tests are concluded in Tab. 1.

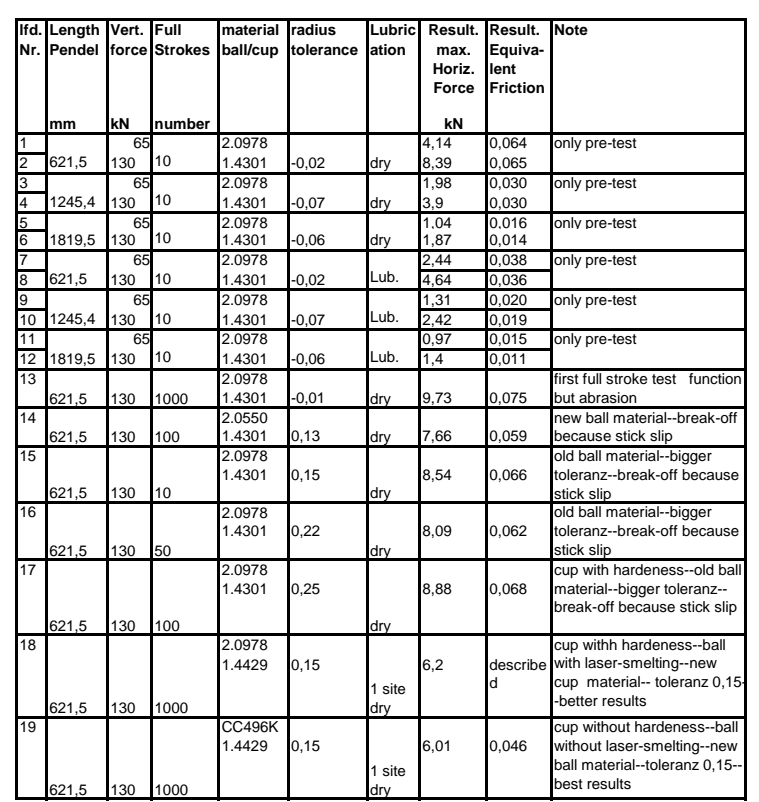

Tab. 1: conclusion of all tests

\section{Tests 1-12 (Pre-Tests):}

Pre-Tests were performed with 3 pendulum supports of different lengths, with 2 different loads. For each test were accomplished 10 strokes lubricated and 10 strokes non-lubricated. 
As expected the horizontal forces were higher with shorter pendulums and no lubrication.

During geometrical measuring it was found out that during the first test campaign the dimension of balls where a little bit larger than the cups. Therefore these tolerances are described in Tab. 3 with "minus".

Of course this cannot work effectively and in the following tests this was changed. This indicates that special attention has to be paid to that during real machining.

\section{Test 13:}

Here where accomplished for the first time 1000 cycles.

The shortest support was used (because of biggest equivalent friction factor) in the non-lubricated state (hardest therms). It was detected that the function is given but the abrasion was high.

(Fig. 6, 7)

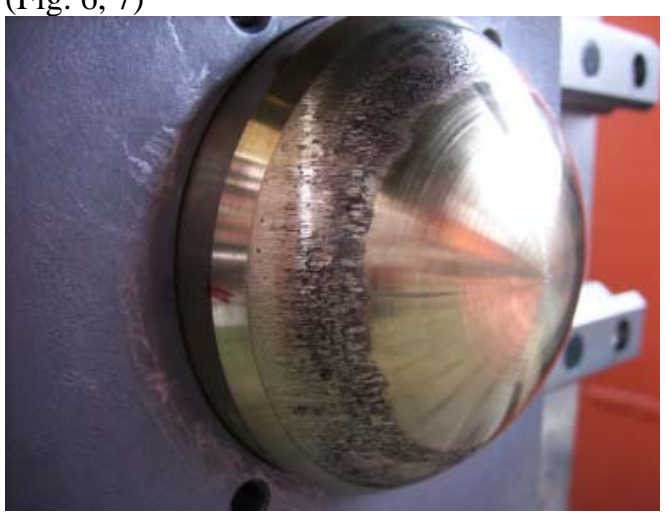

Fig. 6: Test 13/ ball after 1000 strokes

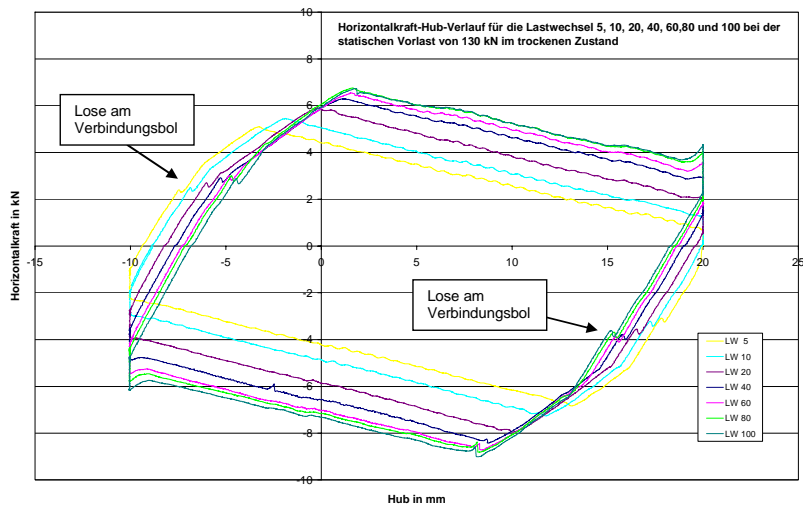

Fig. 7: Test 13/ Graph horizontal Forces/strokes

\section{Tests 14-17:}

These tests where accomplished with different tolerances, ball materials and hardened cups. All these tests were not successful and broken off before 1000 strokes because of Stick Slip.

\section{Test 18:}

There was used cup material 1.4429 (hardened) and ball material laser smelted to reduce Kappa 4 pikes. The radius tolerance was $+0,15 \mathrm{~mm}$ and 1000 strokes were accomplished. Up to now these were the best results. (without Stick Slip and reduced abrasion)

\section{Test 19:}

Here was used again the cup material 1.4429 (but not hardened) and the new ball material CC496K.

This ball material is a plumb-bronze with a plumb content of $15 \%$.

The advantage of this material is the self lubricant system. The compatibility with vacuum is approved.

A problem could be the reduced strength (only up to $120^{\circ} \mathrm{C}$ ), but also this fact is approved in extra tests.

As described before all prior main tests where enforced with the hard request "non lubrication".

In expectance of positive results and to save time and money a new procedure was used in this test.

One side of the pendulum was used again non lubricated and the other lubricated.

The results of this test where the best without Stick Slip, with the lowest equivalent friction factor and the lowest abrasion. (Fig. 8, 9)

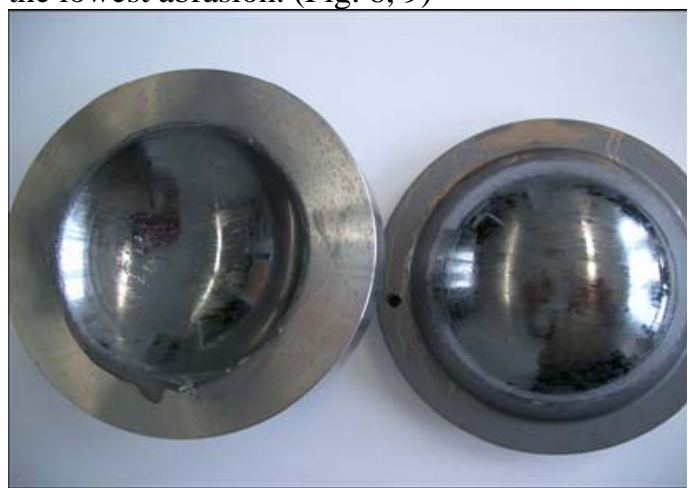

Fig. 8: Test 19/ ball and cup after 1000 strokes

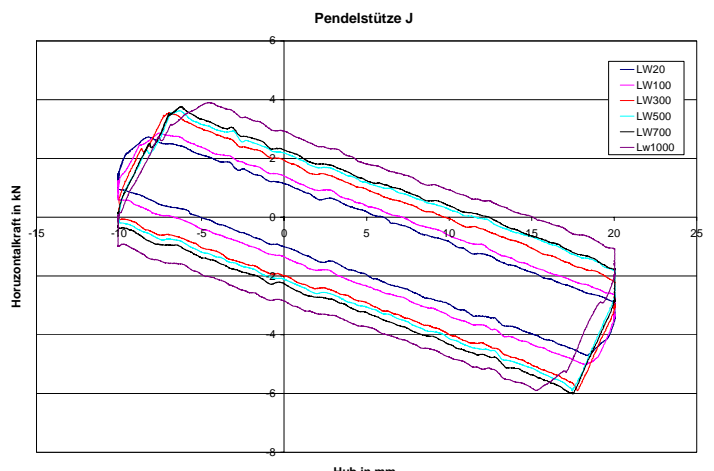

Fig. 9: Test 19/ Graph of horizontal Forces/strokes

\section{Conclusions}

The general feasibility of the pendulum support could be proved with the experiments. Different materials and geometries were tested.

The latest test with cup material 1.4429 and ball material CC496K (plumb-bronze) achieved the best results.

Therefore this will be used for original VVS. The observed smaller horizontal forces triggered by them will be used for following calculations of the whole cryostat. 
5. References

[1] B. Missal, TS Test Pendelstütze für vertikale PG-Abstützung, IPP document Nr. 1ABH00V-S0005.1, 22.12.2005, Max Planck Institut für Plasma- Physik, Greifswald Germany

[2] B. Missal, Zwischen-Auswertung der 1:1 Tests von Pendelstützen (Uni Rostock), IPP document Nr. 1-ABH00V-T0019.0, 12.07.2006, Max Planck Institut für PlasmaPhysik, Greifswald Germany

[3] Dr. P. Friedrich, Abschlussbericht 1:1-Tests von Pendelstützen für die vertikale Abstützung des Plasmagefäßes des Wendelstein 7-X Experimentes, IPP document Nr. 1-ABH00VT2005.2, 02.2007, Universität Rostock, Labor für technische Mechanik I

[4] T. Koppe, Vertical supports of the PV Wendelstein $7-\mathrm{X}$, proc. $24^{\text {th }}$ SOFT, Warshaw $11-15 / 9 / 2006$ 